performance of the machine learning classifiers was compared to a baseline logistic regression model.

Results There were 564 patients in the study population, of whom 307 had a LOS greater than three days and 105 had a LOS greater than seven days. Using the seven-day threshold, the optimal model was the random forest, which achieved an AUC of 0.785 and correctly classified $42.9 \%$ of long LOS patients. Using the three-day threshold, the optimal model was the multilayer perceptron, which achieved an AUC of 0.737 and correctly classified $85.7 \%$ of long LOS patients. The performance of the machine learning models was variable, and they did not unanimously outperform the baseline models.

Conclusions The machine learning models performed poorly in predicting long LOS. Further work is required to assess the clinical utility and value of deep learning methods in an operational setting.

\section{CAPTURING THE INSIGHTS OF ADOLESCENTS WITH LIVED EXPERIENCE OF HEALTH AND SOCIAL CARE TO INFORM THE DESIGN \& DELIVERY OF A TRAINING FOR HSCP THAT SUPPORTS EFFECTIVE CONVERSATIONS WITH CHILDREN \& YOUNG PEOPLE \& THEIR FAMILIES ABOUT TRANSITION}

${ }^{1}$ Lesley Cavalli, ${ }^{2}$ Rachel Naunton, ${ }^{2}$ Rashaun Pacquette-Simpson, ${ }^{2}$ Amy Sutton, ${ }^{3}$ Nigel Mills. ${ }^{1}$ Great Ormond Street Hospital and University College London; ${ }^{2}$ Great Ormond Street Hospital; ${ }^{3}$ Imperial College Healthcare NHS Trust

10.1136/archdischild-2020-gosh.46

Transferring from children's to adult health and social care services can provide both risk and opportunity (Northumbria, 2017). Effective programmes that offer a period of preparation and integration (transition) should be centred on children and young people (CYP) and placed in the context of CYP's lives and should include 'the training of healthcare professionals to utilise effective interpersonal and communication skills' that meet the needs of each CYP (Kime et al 2013, NICE 2016). In 2018 the CQC identified shared decision-making within the top 4 areas requiring improvement in CYP care.

We captured insights from adolescents and their parents with lived experience of transition services both within and external to GOSH. Twenty-nine adolescents participated in an all-day focus group delivered remotely. The group considered 3 key questions. All contributions were transcribed. A separate parent group, focused on the same questions. Themes of Time, Information, Personalisation, Communication and Integration were common to both YP and parents. In addition, YP referenced the impact of mental health on decision making and the impact of health on their education and were aware of diversity and language barriers and the importance of including siblings in communications. Parents had additional concerns around the impact of learning disabilities on their child's transition and of moves to adult learning institutes.

Results provide preliminary information about what 'good should look like' in the design and delivery of our transition services with particular reference to communication. Focus group data, systematic review of the literature and engagement with additional stakeholders including health and social care professionals themselves will be triangulated to develop a coproduced training.

\section{FHIRWORKS 2020: AN INTEROPERABILITY HACKATHON FOR A HEALTHCARE INFORMATION EXCHANGE}

${ }^{1}$ Sheena Visram, ${ }^{2}$ Dean Mohamedally, ${ }^{3}$ Daiana Bassi, ${ }^{3}$ Usman Bahadur, ${ }^{4}$ Costas Stylianou, ${ }^{5} J o n$ MacNamara, ${ }^{6}$ Tom Winstanley, ${ }^{7}$ Glen Quinn, ${ }^{3}$ Neil J Sebire. ${ }^{1}$ University College London; ${ }^{2}$ GOSH DRIVE; ${ }^{3}$ GOSH DRIVE; ${ }^{4}$ Intel; ${ }^{5}$ IBM; ${ }^{6}$ NTT Data; ${ }^{7}$ Aridhia

\subsection{6/archdischild-2020-gosh.47}

Introduction Interoperability in healthcare is a fundamental principle for scalable deployment of novel tools. Fast Healthcare Interoperability Resources (FHIR) comprise data specifications and an application processing interface (API) for the secure exchange of electronic health records (EHR). GOSH DRIVE together with UCL Computer Science and the National Framework of IXNs (Industry Exchange Network) has supported the integration and mainstreaming of standards that make healthcare systems interoperate. Here we present an interoperability hackathon and resulting catalogue of prototypes built using the latest R4 FHIR for healthcare.

Method During February 2020, a two-day hackathon with UCL CS and GOSH DRIVE tasked computer science students with 15 broad challenges to explore FHIR for healthcare. Solutions were built using a synthetic patient record testbed. This testbed offered a FHIR API access allowing connection to any developer environment and technology stack. Mentorship was provided to students by Microsoft, Aridhia, IBM, Intel, EMIS and NTT Data.

Results The GOSH FHIRworks 2020 Hackathon saw 129 FHIR open source demonstrators being built for GOSH DRIVE and the NHS. Exploratory prototypes included smart letter generaters (28), graphical data visualisations (37), and also a breadth of smart watches, scanners, chatbots and AR/ VR demonstrators. This included automated video conferencing over Skype and MS Teams, filtered FHIR data aggregated on Google Maps to visualise patient demographics and immersive solutions that conceptualised patient FHIR records as holograms.

Conclusion As part of the GOSH DRIVE vision to accelerate the deployment of cutting-edge technologies, we have demonstrated effective use of synthetic patient datasets through FHIR APIs to conduct interoperability hackathons. As a next step, these FHIR solutions will be hosted in a demonstrator suite on site to introduce FHIR principles and capabilities to staff at GOSH. We believe that such NHS partnerships with academia facilitate the advancement of safe and secure future healthcare information exchanges.

\section{CHILDREN'S EXPERIENCES OF PERSONAL PROTECTIVE EQUIPMENT (PPE) DURING THE COVID-19 PANDEMIC}

Louise Carter. Anaesthetic Registrar, Great Ormond Street Hospital

\subsection{6/archdischild-2020-gosh.48}

Background The Coronavirus pandemic has affected each one of us from every part of society. From the neonate to centenarians no one is exempt. Our paediatric population has had to adapt rapidly to the huge changes arising from the pandemic, none more so than those coming into hospital and interacting with healthcare professionals. Great Ormond Street Hospital caters for many children with multiple disabilities and/or health problems and rare and congenital conditions. They may present for multiple surgeries and procedures requiring anaesthesia over many years but these interactions 
have had to be very different during 2020. One aspect of the new working model, has been the requirement to wear, 'Personal Protective Equipment.' (PPE). Seeing healthcare workers in full PPE is a new experience for most children and it became obvious, early on during the pandemic, that we needed to find ways to make PPE more,' child-friendly' to minimise anxiety as much as possible for children presenting to hospital and in particular to theatre.

Methods Discussion with colleagues working in the operating theatres at Great Ormond Street Hospital revealed various adaptations to make PPE more, 'child-friendly.' Some children were asked for feedback related to this. A PubMed literature search regarding PPE use in paediatric settings and also the wearing of face masks by children was also conducted. In addition to this, an internet search provided information from other NHS Trusts.

Results Inventive solutions such as cartoon characters on visors and drawing on them have been very well received. Videos about PPE were also helpful. In addition, the importance of non-verbal communication has become very apparent.

Discussion Creating, 'child-friendly,' PPE has been hugely beneficial to children presenting to hospital during the pandemic. It helps to allay their fears and increases their understanding of the current, challenging world that they are living in.

\section{SIGHT ++: PROTOTYPING A COMPUTER VISION GUIDED ASSISTIVE TECHNOLOGY}

${ }^{1}$ Sven Finlay, ${ }^{2}$ Sheena Visram, 'Gisli Georgsson, 'Yanru Chen, 'Songping Lin, 'Xingda Cen, ${ }^{3}$ Costas Stylianou, ${ }^{3}$ Chris Feltham, ${ }^{3}$ Phillippa Chick, ${ }^{1}$ Dean Mohamedally, ${ }^{4}$ Neil J Sebire, ${ }^{1}$ Emmanuel Letier. ${ }^{1}$ UCL Department of Computer Sciences; ${ }^{2}$ University College London; ${ }^{3}$ Intel; ${ }^{4}$ GOSH DRIVE

\subsection{6/archdischild-2020-gosh.49}

Introduction The visually impaired in society are amongst the most impacted by social isolation restrictions of the COVID19 pandemic, fueling research into assistive technologies, including devices primed for computer vision task-orientated image recognition. We present a Proof of Concept prototype modular system that uses Intel RealSense depth cameras connected to a modular ML inference platform to construct near field object information that guides and encourages exploration for users.

Methods Early engagement from experts in the field of global disability enabled us to better appreciate orientation, mobilityrelated considerations, sensory components and meaningful voice instructions. We subsequently designed a novel modular, extensible platform that runs inference classification and depth detection on camera input, then uses heuristic AI Priortiser to analyse and identify essential guidance output for the users.

Results Sight ++ uses object recognition to accurately inform users on near field objects, including data on proximity. By parsing the items through a series of environment rules, which results in objects having more or less relative importance, the system can output qualified audio guidance for obstacle avoidance and awareness. The use of OpenVINO resulted in a 2fold increase in performance of our inference classifiers. We anticipate that a miniaturised depth camera would be fitted to a backpack adjustable strap to offer real-time object recognition and meaningful notification of artefacts of interest at waist height and above.

Conclusion We have produced a robust, foundational assistive system which rather than replace recognised and trusted methods of navigation, introduces a new dimension of intelligence. Future versions will implement motion tracking of the objects, haptic feedback and a teleassistance function connecting a sighted volunteer to enhance guidance. We aspire that future improvements offer a seamless understanding of new environments and create a novel user experience for exploration that are designed for personalisability, social acceptability and social inclusion.

\section{SHAKE, RATTLE AND ROLL - A SERVICE EVALUATION OF A MULTIDISCIPLINARY MUSIC GROUP FOR FAMILIES WITH BERLIN HEARTS}

Zoe Vamplew, Sarah Rickard, Katya Herman, Emma Shkurka. Great Ormond Street Hospital

\subsection{6/archdischild-2020-gosh.50}

Introduction Ventricular assist devices are implanted as a bridge to heart transplant. Inpatient wait time on a Berlin Heart EXCOR (BH), can be up to 17 months. This prolonged hospitalisation has a significant impact on the child's psychological and physiological development, leading to limited educational and social integration. It is important that the developmental treatment of these children incorporates a family centred and holistic approach. This service evaluation assessed the effectiveness and safety of a multidisciplinary music group on $\mathrm{BH}$ patients, their carers and nursing staff.

Method A structured multidisciplinary music group was created by Physiotherapy, Music and Occupational therapy. Carer involvement was a key component. Each session included: singing, actions, turn taking, gross and fine motor activities and a story. Feedback via anonymous survey was sought from carers and nursing staff after six sessions regarding structure, content and environment.

Results Five children and six carers participated over 12 weeks. The average age was 21 months and wait time since implantation 126-368 days. All carers and three bedside nurses completed the survey.

$75 \%$ carers found the group beneficial and $75 \%$ felt their child enjoyed all components. Carers reported improved peer interaction and motor development. All advocated for the group to continue. Feedback included more actions and sensory stories. All nurses found the group overall 'extremely beneficial' and $66.67 \%$ felt the children 'extremely enjoyed' the sessions. $100 \%$ observed positive changes in the children and carers since commencement. No adverse events or safety concerns were raised.

Conclusion This service evaluation suggests that the multidisciplinary music group is a safe and valuable therapeutic component that can improve family experience and interaction for children on a $\mathrm{BH}$. Carers and staff observed a positive impact on motor and social development as well as enjoyment. The group is now routinely part of the $\mathrm{BH}$ therapy programme.

\section{POSCU - JOINED UP WORKING VIA GOSHLINK}

Bing Wang, Lynne Riley, Rachel Edmead, Joshua Giddings, Chris Grant. Great Ormond Street Hospital

\subsection{6/archdischild-2020-gosh.51}

All members of the patient's care team using GOSHLink can view the patient's full, contemporaneous electronic medical 